\title{
Some new results on sequence spaces with respect to non-Newtonian calculus
}

\author{
Ahmet Faruk Çakmak and Feyzi Başar ${ }^{2 *}$
}

"Correspondence:

fbasar@fatih.edu.tr;

feyzibasar@gmail.com

${ }^{2}$ Department of Mathematics,

Faculty of Arts and Sciences, Fatih University, The Hadımköy Campus,

Büyükçekmece, İstanbul, 34500,

Turkey

Full list of author information is

available at the end of the article

\begin{abstract}
As an alternative to classical calculus, Grossman and Katz (Non-Newtonian Calculus, 1972) introduced the non-Newtonian calculus consisting of the branches of geometric, anageometric and bigeometric calculus etc. Following Grossman and Katz, we construct the field $\mathbb{R}(N)$ of non-Newtonian real numbers and the concept of non-Newtonian metric. Also, we give the triangle and Minkowski's inequalities in the sense of non-Newtonian calculus. Later, we respectively define the sets $\omega(N), \ell_{\infty}(N)$, $c(N), c_{0}(N)$ and $\ell_{p}(N)$ of all, bounded, convergent, null and $p$-absolutely summable sequences in the sense of non-Newtonian calculus and show that each of the sets forms a vector space on the field $\mathbb{R}(N)$ and a complete metric space.
\end{abstract}

MSC: 26A06; 11U10; 08A05

Keywords: non-Newtonian calculus; algebraic structures with respect to non-Newtonian calculus; non-Newtonian inequalities; non-Newtonian sequence spaces

\section{Preliminaries, background and notations}

During the Renaissance many scholars, including Galileo, discussed the following problem: Two estimates, 10 and 1,000, are proposed as the value of a horse. Which estimate, if any, deviates more from the true value of 100 ? The scholars who maintained that deviations should be measured by differences concluded that the estimate of 10 was closer to the true value. However, Galileo eventually maintained that the deviations should be measured by ratios and concluded that two estimates deviated equally from the true value (cf. [1]).

From the story, the question comes out this way: What if we measure by ratios? The answer is the main idea of non-Newtonian calculus which consists of many calculi such as classical, geometric, anageometric, bigeometric calculus etc. Even you can create your own calculus by choosing a different function as a generator. Although all arithmetics are structurally equivalent, only by distinguishing among them do we obtain suitable tools for constructing all the non-Newtonian calculi. But the usefulness of arithmetics is not limited to the construction of calculus; we believe there is a more fundamental reason for considering alternative arithmetics. They may also be helpful in developing and understanding the new systems of measurement that could yield simpler physical laws (cf. [1]).

Bashirov et al. [2] have recently concentrated on multiplicative calculus and gave the results with applications corresponding to the well-known properties of derivatives and integrals in classical calculus. Quite recently, Uzer [3] has extended the multiplicative cal-

(c) 2012 Cakmak and Başar; licensee Springer. This is an Open Access article distributed under the terms of the Creative Commons Attribution License (http://creativecommons.org/licenses/by/2.0), which permits unrestricted use, distribution, and reproduction in any medium, provided the original work is properly cited. 
culus to the complex valued functions and was interested in the statements of some fundamental theorems and concepts of multiplicative complex calculus, and demonstrated some analogies between the multiplicative complex calculus and classical calculus by theoretical and numerical examples.

Non-Newtonian calculus is an alternative to the usual calculus of Newton and Leibniz. It provides differentiation and integration tools based on non-Newtonian operations instead of classical operations. Every property in classical calculus has an analogue in nonNewtonian calculus. Generally speaking, non-Newtonian calculus is a methodology that allows one to have a different look at problems which can be investigated via calculus. In some cases, for example, for wage-rate (in dollars, euro etc.) related problems, the use of bigeometric calculus, which is a kind of non-Newtonian calculus, is advocated instead of the traditional Newtonian one (cf. [1]).

Throughout this document, non-Newtonian calculus is denoted by (NC), and classical calculus is denoted by (CC). A generator is a one-to-one function whose domain is $\mathbb{R}$ and whose range is a subset of $\mathbb{R}$, the set of real numbers. Each generator generates exactly one arithmetic and, conversely, each arithmetic is generated by exactly one generator. As a generator, we choose the function exp from $\mathbb{R}$ to the set $\mathbb{R}^{+}$of positive reals, that is to say,

$$
\begin{aligned}
\alpha: \mathbb{R} & \longrightarrow \mathbb{R}^{+} \\
x & \longmapsto \alpha(x)=e^{x}=y
\end{aligned} \text { and } \begin{aligned}
\alpha^{-1}: \mathbb{R}^{+} & \longrightarrow \mathbb{R} \\
y & \longmapsto \alpha^{-1}(y)=\ln y=x .
\end{aligned}
$$

If $I(x)=x$ for all $x \in \mathbb{R}$, then $I$ is called identity function whose inverse is itself. In the special cases $\alpha=I$ and $\alpha=\exp , \alpha$ generates the classical and geometric arithmetics, respectively. The set $\mathbb{R}(N)$ of non-Newtonian real numbers is defined as $\mathbb{R}(N):=\{\alpha(x): x \in \mathbb{R}\}$.

Following Bashirov et al. [2], Uzer [3] and Türkmen and Başar [4], the main purpose of this paper is to construct the classical sequence spaces with respect to non-Newtonian calculus over the non-Newtonian real field. To do this, we need some preparatory knowledge about non-Newtonian calculus.

In the foundations of science (formerly titled Physics: The Elements), Norman Robert Campbell, a pioneer in the theory of measurement, clearly recognized that alternative arithmetics might be useful in science for he wrote, 'We must recognize the possibility that a system of measurement may be arbitrary otherwise than in the choice of unit; there may be arbitrariness in the choice of process of addition' ( $c f$. [1]).

We should know that all concepts in classical arithmetic have natural counterparts in $\alpha$-arithmetic. For instance, $\alpha$-zero and $\alpha$-one turn out to be $\alpha(0)$ and $\alpha(1)$. Similarly, the $\alpha$-integers turn out to be the following:

$$
\ldots, \alpha(-3), \alpha(-2), \alpha(-1), \alpha(0), \alpha(1), \alpha(2), \alpha(3), \ldots
$$

Consider any generator $\alpha$ with range $A$. By $\alpha$-arithmetic, we mean the arithmetic whose domain is $A$ and whose operations are defined as follows: For $x, y \in \mathbb{R}$ and any generator $\alpha$,

$$
\begin{array}{ll}
\alpha \text {-addition } & x \dot{+} y=\alpha\left\{\alpha^{-1}(x)+\alpha^{-1}(y)\right\}, \\
\alpha \text {-subtraction } & x \dot{-} y=\alpha\left\{\alpha^{-1}(x)-\alpha^{-1}(y)\right\}, \\
\alpha \text {-multiplication } & x \dot{\times} y=\alpha\left\{\alpha^{-1}(x) \times \alpha^{-1}(y)\right\}, \\
\alpha \text {-division } & x \dot{y} y=\alpha\left\{\alpha^{-1}(x) \div \alpha^{-1}(y)\right\}, \\
\alpha \text {-order } & x \dot{<} y \Leftrightarrow \alpha^{-1}(x)<\alpha^{-1}(y) .
\end{array}
$$


Particularly, if we choose $\alpha$-generator $I$, the identity function $\alpha(x)=x$ for all $x \in \mathbb{R} \Rightarrow$ $\alpha^{-1}(x)=x$, then obviously $\alpha$-arithmetic turns out to be classical arithmetic.

$$
\begin{array}{lrl}
\alpha \text {-addition } & x \oplus y & =\alpha\left\{\alpha^{-1}(x)+\alpha^{-1}(y)\right\}=\alpha\{x+y\}=x+y \text { classic addition, } \\
\alpha \text {-subtraction } & x \ominus y & =\alpha\left\{\alpha^{-1}(x)-\alpha^{-1}(y)\right\}=\alpha\{x-y\}=x-y \text { classic subtraction, } \\
\alpha \text {-multiplication } & x \odot y & =\alpha\left\{\alpha^{-1}(x) \times \alpha^{-1}(y)\right\}=\alpha\{x \times y\} \\
& =x \times y \text { classic multiplication, } \\
\alpha \text {-division } & x \oslash y & =\alpha\left\{\alpha^{-1}(x) \div \alpha^{-1}(y)\right\}=\alpha\{x \div y\} \\
& =x \div y, y \neq 0 \text { classic division. }
\end{array}
$$

If we choose $\alpha$-generator as $\exp , \alpha(z)=e^{z}$ for $z \in \mathbb{C}$, then $\alpha^{-1}(z)=\ln z, \alpha$-arithmetic turns out to be geometric arithmetic.

$$
\begin{aligned}
& \alpha \text {-addition } \quad x \oplus y=\alpha\left\{\alpha^{-1}(x)+\alpha^{-1}(y)\right\}=e^{\{\ln x+\ln y\}}=x \cdot y \text { geometric addition, } \\
& \alpha \text {-subtraction } \quad x \ominus y=\alpha\left\{\alpha^{-1}(x)-\alpha^{-1}(y)\right\}=e^{\{\ln x-\ln y\}} \\
& =x \div y, y \neq 0 \text { geometric subtraction, } \\
& \alpha \text {-multiplication } \quad x \odot y=\alpha\left\{\alpha^{-1}(x) \times \alpha^{-1}(y)\right\}=e^{\{\ln x \times \ln y\}} \\
& =x^{\ln y}=y^{\ln x} \text { geometric multiplication, } \\
& \alpha \text {-division } \quad x \oslash y=\alpha\left\{\alpha^{-1}(x) \div \alpha^{-1}(y)\right\}=e^{\{\ln x \div \ln w\}} \\
& =x^{\frac{1}{\ln y}}, y \neq 1 \text { geometric division. }
\end{aligned}
$$

An arithmetic is any system that satisfies the complete ordered field axioms and has a domain that is a subset of $\mathbb{R}$. There are infinitely many arithmetics, all of which are isomorphic, that is, structurally equivalent. Nevertheless, the fact that two systems are isomorphic does not preclude their separate uses. In [1], it is shown that each ordered pair of arithmetics gives rise to a calculus by a judicious use of the first arithmetic for function arguments and the second arithmetic for function values. Table 1 indicates the four calculi obtainable by using the classical and geometric arithmetics.

The rest of the paper is organized, as follows. In Section 2, it is shown that the set $\mathbb{R}(N)$ of non-Newtonian real numbers forms a field with the binary operations addition $(\dot{+})$ and $(\dot{x})$. Further, the non-Newtonian exponent, surd and absolute value are defined and some of their properties are given. Section 3 is devoted to the vector space over the field $\mathbb{R}(N)$ of non-Newtonian real numbers. Additionally, some required inequalities are presented in the sense of non-Newtonian calculus, and the concepts of non-Newtonian metric and non-Newtonian norm are introduced. In Section 4, subsequent to giving the corresponding results for the sequences of non-Newtonian real numbers concerning the convergent sequences of real numbers, it is stated and proved that the $n$-dimensional non-Newtonian real space $\mathbb{R}^{n}(N)$ is a complete metric space. In Section 5 , prior to showing the sets $\omega(N)$, $\ell_{\infty}(N), c(N), c_{0}(N)$ and $\ell_{p}(N)$ of all, bounded, convergent, null and absolutely $p$-summable sequences of the non-Newtonian real numbers are the complete metric spaces, it is proved

Table 1 Four calculi obtained by using classical and geometric arithmetic

\begin{tabular}{lll}
\hline Name of calculus & First arithmetics (arguments) & Second arithmetics (values) \\
\hline Classical calculus & Classical & Classical \\
Bigeometric calculus & Geometric & Geometric \\
Geometric calculus & Classical & Geometric \\
Anageometric calculus & Geometric & Classical \\
\hline
\end{tabular}


that each of those sets forms a vector space over the field $\mathbb{R}(N)$ with respect to the algebraic operations addition $(\dot{+})$ and scalar multiplication $(\dot{x})$. In the final section of the paper, we note the significance of non-Newtonian calculus and record some further suggestions.

\section{Non-Newtonian real field and related properties}

In the present section, we construct the non-Newtonian real field $\mathbb{R}(N)$ and give some related properties.

We define the binary operations $(\dot{+})$ addition and $(\dot{x})$ multiplication for the set $\mathbb{R}(N)$ of non-Newtonian real numbers by

$$
\begin{aligned}
\dot{+}: \mathbb{R} \times \mathbb{R} & \longrightarrow \mathbb{R} \\
(x, y) & \mapsto x+y=\alpha\left\{\alpha^{-1}(x)+\alpha^{-1}(y)\right\} \quad \text { and } \\
\dot{\times}: \mathbb{R} \times \mathbb{R} & \longrightarrow \mathbb{R} \\
(x, y) & \mapsto x \dot{\times} y=\alpha\left\{\alpha^{-1}(x) \times \alpha^{-1}(y)\right\} .
\end{aligned}
$$

Theorem $2.1(\mathbb{R}(N), \dot{+}, \dot{x})$ is a complete field.

Proof One can show this by a routine verification, so we omit the detail.

The $\alpha$-square of a number $x$ in $A \subset \mathbb{R}(N)$ is denoted by $x \dot{\times} x$ which will be denoted by $x^{2}$. For each $\alpha$-nonnegative number $t$, the symbol $\sqrt{x}^{N}$ will be used to denote $t=$ $\alpha\left\{\sqrt{\alpha^{-1}(x)}\right\}$ which is the unique $\alpha$-nonnegative number whose $\alpha$-square is equal to $x$, which means $t^{2}=x$.

Throughout this section, we denote the $p$ th non-Newtonian exponent and the $q$ th nonNewtonian root of $x \in \mathbb{R}(N)$ by $x^{p_{N}}$ and $\sqrt[q]{x}{ }^{N}$, respectively. Therefore, we have

$$
\begin{aligned}
x^{2 N}=x \dot{\times} x & =\alpha\left\{\alpha^{-1}(x) \times \alpha^{-1}(x)\right\}=\alpha\left\{\left[\alpha^{-1}(x)\right]^{2}\right\}, \\
x^{3_{N}}=x^{2_{N}} \dot{\times} x & =\alpha\left\{\alpha^{-1}\left\{\alpha\left[\alpha^{-1}(x) \times \alpha^{-1}(x)\right]\right\} \times \alpha^{-1}(x)\right\}=\alpha\left\{\left[\alpha^{-1}(x)\right]^{3}\right\}, \\
& \vdots \\
x^{p_{N}}=x^{(p-1)_{N}} \dot{\times} x & =\alpha\left\{\left[\alpha^{-1}(x)\right]^{p}\right\},
\end{aligned}
$$

The $\alpha$-absolute value of a number $x$ in $A \subset \mathbb{R}(N)$ is defined as $\alpha\left(\left|\alpha^{-1}(x)\right|\right)$ and is denoted by $|x|_{N}$. For each number $x$ in $A \subset \mathbb{R}(N)$,

$$
{\sqrt{x^{2}}}^{N}=|x|_{N}=\alpha\left(\left|\alpha^{-1}(x)\right|\right) .
$$

Then we say,

$$
|x|_{N}= \begin{cases}x, & x>\alpha(0), \\ \alpha(0), & x=\alpha(0)=\alpha\left\{\left|\alpha^{-1}(x)\right|\right\} \\ \alpha(0) \dot{-} x, & x<\alpha(0) .\end{cases}
$$


The non-Newtonian distance between two numbers $x_{1}$ and $x_{2}$ is defined by

$$
\begin{aligned}
\left|x_{1} \dot{-} x_{2}\right|_{N} & =\alpha\left\{\left|\alpha^{-1}\left(x_{1}\right)-\alpha^{-1}\left(x_{2}\right)\right|\right\} \\
& =\alpha\left\{\left|\alpha^{-1}\left(x_{2}\right)-\alpha^{-1}\left(x_{1}\right)\right|\right\} \\
& =\left|x_{2} \dot{-} x_{1}\right|_{N} .
\end{aligned}
$$

Let any $z \in \mathbb{R}(N)$ be given. Then $z$ is called a positive non-Newtonian real number if $z \dot{>} \alpha(0)$ and $z$ is called a non-Newtonian negative real number if $z \dot{<} \alpha(0)$, and finally, $z$ is called an unsigned non-Newtonian real number if $z=\alpha(0)$. By $\mathbb{R}^{+}(N)$ and $\mathbb{R}^{-}(N)$, we denote the sets of non-Newtonian positive and negative real numbers, respectively.

In classical calculus, we have $|x y|=|x||y|$ for $x, y \in \mathbb{R}$. The following proposition shows that this also holds in non-Newtonian calculus.

Proposition 2.2 For any $x, y \in \mathbb{R}(N),|x \dot{\times} y|_{N}=|x|_{N} \dot{x}|y|_{N}$.

Proof By using the definition of the binary operation $(\dot{x})$, one can easily see that

$$
\begin{aligned}
|x \dot{x} y|_{N} & =\alpha\left[\left|\alpha^{-1}(x) \times \alpha^{-1}(y)\right|\right] \\
& =\alpha\left[\left|\alpha^{-1}(x)\right| \times\left|\alpha^{-1}(y)\right|\right] \\
& =\alpha\left[\left|\alpha^{-1}(x)\right|\right\} \dot{\times} \alpha\left\{\left|\alpha^{-1}(y)\right|\right] \\
& =|x|_{N} \dot{\times}|y|_{N},
\end{aligned}
$$

as desired.

\section{Non-Newtonian metric spaces}

In this section, we deal with the vector spaces over the non-Newtonian real field $\mathbb{R}(N)$. First, we present the required inequalities in the sense of (NC).

Lemma 3.1 (Triangle inequality) Let $x, y \in \mathbb{R}(N)$. Then

$$
|x \dot{+} y|_{N} \leq|x|_{N} \dot{+}|y|_{N} .
$$

Proof Let $x, y \in \mathbb{R}(N)$. Taking into account the definition of absolute value given by (2.1), one can see that

$$
\begin{aligned}
|x+y|_{N} & =\alpha\left[\left|\alpha^{-1}(x+y)\right|\right] \\
& =\alpha\left\{\left|\left(\alpha^{-1} \circ \alpha\right)\left[\alpha^{-1}(x)+\alpha^{-1}(y)\right]\right|\right\} \\
& =\alpha\left[\left|\alpha^{-1}(x)+\alpha^{-1}(y)\right|\right] .
\end{aligned}
$$

Therefore, we obtain by applying $\alpha^{-1}$ to the equality (3.2) that

$$
\begin{aligned}
\alpha^{-1}\left(|x \dot{x} y|_{N}\right) & =\left|\alpha^{-1}(x)+\alpha^{-1}(y)\right| \\
& \leq\left|\alpha^{-1}(x)\right|+\left|\alpha^{-1}(y)\right|,
\end{aligned}
$$


which leads us to

$$
\begin{aligned}
|x \dot{+} y|_{N} & \leq \alpha\left[\left|\alpha^{-1}(x)\right|+\left|\alpha^{-1}(y)\right|\right] \\
& =\alpha\left\{\left(\alpha^{-1} \circ \alpha\right)\left[\left|\alpha^{-1}(x)\right|\right]+\left(\alpha^{-1} \circ \alpha\right)\left[\left|\alpha^{-1}(y)\right|\right]\right\} \\
& =\alpha\left[\alpha^{-1}\left(|x|_{N}\right)+\alpha^{-1}\left(|y|_{N}\right)\right] \\
& =|x|_{N} \dot{+}|y|_{N} .
\end{aligned}
$$

This shows that (3.1) holds.

Lemma 3.2 Let $u, v \in \mathbb{R}(N)$. Then

$$
\frac{|u \dot{+} v|_{N}}{\alpha(1) \dot{+}|u \dot{+} v|_{N}} N \dot{\leq} \frac{|u|_{N}}{\alpha(1) \dot{+}|u|_{N}} N \dot{+} \frac{|v|_{N}}{\alpha(1) \dot{+}|v|_{N}} N .
$$

Proof Let $z$ be

$$
\begin{aligned}
z & =\frac{|u \dot{+} v|_{N}}{\alpha(1) \dot{+}|u \dot{+} v|_{N}} N=\frac{\left|\alpha\left[\alpha^{-1}(u)+\alpha^{-1}(v)\right]\right|_{N}}{\alpha(1) \dot{+}\left|\alpha\left[\alpha^{-1}(u)+\alpha^{-1}(v)\right]\right|_{N}} N \\
& =\frac{\alpha\left\{\left|\alpha^{-1}\left(\alpha\left[\alpha^{-1}(u)+\alpha^{-1}(v)\right]\right)\right|\right\}}{\alpha(1) \dot{+} \alpha\left\{\left|\alpha^{-1}\left(\alpha\left[\alpha^{-1}(u)+\alpha^{-1}(v)\right]\right)\right|\right\}} N \\
& =\frac{\alpha\left\{\left|\alpha^{-1}(u)+\alpha^{-1}(v)\right|\right\}}{\alpha\left\{\alpha^{-1}(\alpha(1))+\alpha^{-1}\left(\alpha\left\{\left|\alpha^{-1}(u)+\alpha^{-1}(v)\right|\right\}\right)\right\}} N \\
& =\frac{\alpha\left\{\left|\alpha^{-1}(u)+\alpha^{-1}(v)\right|\right\}}{\alpha\left\{1+\left|\alpha^{-1}(u)+\alpha^{-1}(v)\right|\right\}} N \\
& =\frac{\phi}{\psi} N=\alpha\left\{\alpha^{-1}(\phi) / \alpha^{-1}(\psi)\right\} \\
& =\frac{\alpha\left\{\left(\alpha^{-1} \circ \alpha\right)\left[\left|\alpha^{-1}(u)+\alpha^{-1}(v)\right|\right]\right\}}{\left(\alpha^{-1} \circ \alpha\right)\left[1+\left|\alpha^{-1}(u)+\alpha^{-1}(v)\right|\right]} \\
& =\alpha\left\{\frac{\left|\alpha^{-1}(u)+\alpha^{-1}(v)\right|}{\left[1+\left|\alpha^{-1}(u)+\alpha^{-1}(v)\right|\right]}\right\} .
\end{aligned}
$$

Therefore, one can easily observe by applying $\alpha^{-1}$ to the equality (3.4) that

$$
\begin{aligned}
\alpha^{-1}(z) & =\frac{\left|\alpha^{-1}(u)+\alpha^{-1}(v)\right|}{1+\left|\alpha^{-1}(u)+\alpha^{-1}(v)\right|} \\
& \leq \frac{\left|\alpha^{-1}(u)\right|}{1+\left|\alpha^{-1}(u)\right|}+\frac{\left|\alpha^{-1}(v)\right|}{1+\left|\alpha^{-1}(v)\right|} .
\end{aligned}
$$

Then

$$
\begin{aligned}
z & \leq \alpha\left[\frac{\left|\alpha^{-1}(u)\right|}{1+\left|\alpha^{-1}(u)\right|}+\frac{\left|\alpha^{-1}(v)\right|}{1+\left|\alpha^{-1}(v)\right|}\right] \\
& =\alpha\left[\frac{\left(\alpha^{-1} \circ \alpha\right)\left|\alpha^{-1}(u)\right|}{1+\left(\alpha^{-1} \circ \alpha\right)\left|\alpha^{-1}(u)\right|}+\frac{\left(\alpha^{-1} \circ \alpha\right)\left|\alpha^{-1}(v)\right|}{1+\left(\alpha^{-1} \circ \alpha\right)\left|\alpha^{-1}(v)\right|}\right] \\
& =\alpha\left\{\frac{\alpha^{-1}\left[\alpha\left(\left|\alpha^{-1}(u)\right|\right)\right]}{1+\alpha^{-1}\left[\alpha\left(\left|\alpha^{-1}(u)\right|\right)\right]}+\frac{\alpha^{-1}\left[\alpha\left(\left|\alpha^{-1}(v)\right|\right)\right]}{1+\alpha^{-1}\left[\alpha\left(\left|\alpha^{-1}(v)\right|\right)\right]}\right\}
\end{aligned}
$$




$$
\begin{aligned}
& =\alpha\left[\frac{\alpha^{-1}\left(|u|_{N}\right)}{1+\alpha^{-1}\left(|u|_{N}\right)}\right]+\alpha\left[\frac{\alpha^{-1}\left(|v|_{N}\right)}{1+\alpha^{-1}\left(|v|_{N}\right)}\right] \\
& =\alpha\left[\frac{\alpha^{-1}\left(|u|_{N}\right)}{\left(\alpha^{-1} \circ \alpha\right)(1)+\alpha^{-1}\left(|u|_{N}\right)}\right]+\alpha\left[\frac{\alpha^{-1}\left(|v|_{N}\right)}{\left(\alpha^{-1} \circ \alpha\right)(1)+\alpha^{-1}\left(|v|_{N}\right)}\right] \\
& =\alpha\left\{\frac{\alpha^{-1}\left(|u|_{N}\right)}{\alpha^{-1}[\alpha(1)]+\alpha^{-1}\left(|u|_{N}\right)}\right\}+\alpha\left\{\frac{\alpha^{-1}\left(|v|_{N}\right)}{\alpha^{-1}[\alpha(1)]+\alpha^{-1}\left(|v|_{N}\right)}\right\} \\
& =\alpha\left\{\frac{\alpha^{-1}\left(|u|_{N}\right)}{\left(\alpha^{-1} \circ \alpha\right) \alpha^{-1}(\alpha(1))+\alpha^{-1}\left(|u|_{N}\right)}\right\}+\alpha\left\{\frac{\alpha^{-1}\left(|v|_{N}\right)}{\left(\alpha^{-1} \circ \alpha\right) \alpha^{-1}(\alpha(1))+\alpha^{-1}\left(|v|_{N}\right)}\right\} \\
& =\alpha\left\{\frac{\alpha^{-1}\left(|u|_{N}\right)}{\left(\alpha^{-1} \circ \alpha\right)\left\{\alpha^{-1}[\alpha(1)]+\alpha^{-1}\left(|u|_{N}\right)\right\}}+\frac{\alpha^{-1}\left(|v|_{N}\right)}{\left(\alpha^{-1} \circ \alpha\right)\left\{\alpha^{-1}[\alpha(1)]+\alpha^{-1}\left(|v|_{N}\right)\right\}}\right\} \\
& =\alpha\left\{\frac{\alpha^{-1}\left(|u|_{N}\right)}{\alpha^{-1}\left[\alpha(1) \dot{+}|u|_{N}\right]}+\frac{\alpha^{-1}\left(|v|_{N}\right)}{\alpha^{-1}\left[\alpha(1) \dot{+}|v|_{N}\right]}\right\} \\
& =\alpha\left\{\left(\alpha^{-1} \circ \alpha\right)\left\{\frac{\alpha^{-1}\left(|u|_{N}\right)}{\alpha^{-1}\left[\alpha(1) \dot{+}|u|_{N}\right]}\right\}+\left(\alpha^{-1} \circ \alpha\right)\left\{\frac{\alpha^{-1}\left(|v|_{N}\right)}{\alpha^{-1}\left[\alpha(1) \dot{+}|v|_{N}\right]}\right\}\right\} \\
& =\alpha\left\{\alpha^{-1}\left[\frac{|u|_{N}}{\alpha(1) \dot{+}|u|_{N}} N\right]+\alpha^{-1}\left[\frac{|v|_{N}}{\alpha(1) \dot{+}|v|_{N}} N\right]\right\} \\
& =\frac{|u|_{N}}{\alpha(1) \dot{+}|u|_{N}} N \dot{+} \frac{|v|_{N}}{\alpha(1) \dot{+}|v|_{N}} N,
\end{aligned}
$$

which completes the proof.

Lemma 3.3 (Minkowski's inequality) Let $p>1$ and $a_{k}, b_{k} \in \mathbb{R}^{+}(N)$ for $k \in\{1,2,3, \ldots, n\}$.

Then

$$
\sqrt[p]{\sum_{N} \sum_{k=1}^{n}\left(a_{k} \dot{+} b_{k}\right)^{p_{N}}} \dot{-}{\sqrt[p]{\sum_{k=1}^{n} a_{k} p_{N}}}^{N}+\sqrt[p]{\sum_{N}^{\sum_{k=1}^{n} b_{k}^{p_{N}}}} .
$$

Proof Suppose that

$$
u=\sqrt[p]{\sum_{N} \sum_{k=1}^{n}\left(a_{k}+b_{k}\right)^{p_{N}}}=\left\{\sum_{N} \sum_{k=1}^{n}\left\{\alpha\left[\alpha^{-1}\left(a_{k}\right)+\alpha^{-1}\left(b_{k}\right)\right]\right\}^{p_{N}}\right\}^{(1 / p)_{N}} .
$$

Then we have

$$
\begin{aligned}
u^{p_{N}} & ={ }_{N} \sum_{k=1}^{n}\left\{\alpha\left[\alpha^{-1}\left(a_{k}\right)+\alpha^{-1}\left(b_{k}\right)\right]\right\}^{p_{N}} \\
& =\sum_{N}^{n} \alpha\left\{\left[\alpha^{-1}\left(\alpha\left\{\alpha^{-1}\left(a_{k}\right)+\alpha^{-1}\left(b_{k}\right)\right\}\right)\right]^{p}\right\} \\
& ={ }_{N} \sum_{k=1}^{n} \alpha\left\{\left[\alpha^{-1}\left(a_{k}\right)+\alpha^{-1}\left(b_{k}\right)\right]^{p}\right\} \\
& =\alpha\left\{\alpha^{-1}\left(\alpha\left\{\left[\alpha^{-1}\left(a_{1}\right)+\alpha^{-1}\left(b_{1}\right)\right]^{p}\right\}\right)+\cdots+\alpha^{-1}\left(\alpha\left\{\left[\alpha^{-1}\left(a_{n}\right)+\alpha^{-1}\left(b_{n}\right)\right]^{p}\right\}\right)\right\} \\
& =\alpha\left\{\left[\alpha^{-1}\left(a_{1}\right)+\alpha^{-1}\left(b_{1}\right)\right]^{p}+\cdots+\left[\alpha^{-1}\left(a_{n}\right)+\alpha^{-1}\left(b_{n}\right)\right]^{p}\right\}
\end{aligned}
$$




$$
\begin{aligned}
=\alpha\left\{\sum_{k=1}^{n}\left[\alpha^{-1}\left(a_{k}\right)+\alpha^{-1}\left(b_{k}\right)\right]^{p}\right\} \\
\alpha^{-1}\left(u^{p_{N}}\right)=\sum_{k=1}^{n}\left[\alpha^{-1}\left(a_{k}\right)+\alpha^{-1}\left(b_{k}\right)\right]^{p}, \\
{\left[\alpha^{-1}\left(u^{p_{N}}\right)\right]^{1 / p}=\left\{\sum_{k=1}^{n}\left[\alpha^{-1}\left(a_{k}\right)+\alpha^{-1}\left(b_{k}\right)\right]^{p}\right\}^{1 / p} } \\
\leq\left\{\sum_{k=1}^{n}\left[\alpha^{-1}\left(a_{k}\right)\right]^{p}\right\}^{1 / p}+\left\{\sum_{k=1}^{n}\left[\alpha^{-1}\left(b_{k}\right)\right]^{p}\right\}^{1 / p} .
\end{aligned}
$$

Therefore, one can easily observe by applying $\alpha$ to the equality (3.5) with Minkowski's inequality that

$$
\begin{aligned}
& \alpha\left\{\left[\alpha^{-1}\left(u^{p_{N}}\right)\right]^{1 / p}\right\} \\
& \dot{\leq} \alpha\left\{\left[\sum_{k=1}^{n}\left[\alpha^{-1}\left(a_{k}\right)\right]^{p}\right]^{1 / p}+\left[\sum_{k=1}^{n}\left[\alpha^{-1}\left(b_{k}\right)\right]^{p}\right]^{1 / p}\right\} \\
& =\alpha\left\{\left(\alpha^{-1} \circ \alpha\right)\left\{\sum_{k=1}^{n}\left[\alpha^{-1}\left(a_{k}\right)\right]^{p}\right\}^{1 / p}+\left(\alpha^{-1} \circ \alpha\right)\left\{\sum_{k=1}^{n}\left[\alpha^{-1}\left(b_{k}\right)\right]^{p}\right\}^{1 / p}\right\} \\
& =\alpha\left\{\left[\sum_{k=1}^{n}\left[\alpha^{-1}\left(a_{k}\right)\right]^{p}\right]^{1 / p}\right\} \dot{+} \alpha\left\{\left[\sum_{k=1}^{n}\left[\alpha^{-1}\left(b_{k}\right)\right]^{p}\right]^{1 / p}\right\} \\
& =\alpha\left\{\left[\sum_{k=1}^{n}\left(\alpha^{-1} \circ \alpha\right)\left[\alpha^{-1}\left(a_{k}\right)\right]^{p}\right]^{1 / p}\right\} \dot{+} \alpha\left\{\left[\sum_{k=1}^{n}\left(\alpha^{-1} \circ \alpha\right)\left[\alpha^{-1}\left(b_{k}\right)\right]^{p}\right]^{1 / p}\right\} \\
& =\alpha\left\{\left[\sum_{k=1}^{n} \alpha^{-1}\left(a_{k}^{p_{N}}\right)\right]^{1 / p}\right\} \dot{+} \alpha\left\{\left[\sum_{k=1}^{n} \alpha^{-1}\left(b_{k}^{p_{N}}\right)\right]^{1 / p}\right\} \\
& =\alpha\left\{\left(\alpha^{-1} \circ \alpha\right)\left[\sum_{k=1}^{n} \alpha^{-1}\left(a_{k}^{p_{N}}\right)\right]^{1 / p}\right\} \dot{+} \alpha\left\{\left(\alpha^{-1} \circ \alpha\right)\left[\sum_{k=1}^{n} \alpha^{-1}\left(b_{k}^{p_{N}}\right)\right]^{1 / p}\right\} \\
& =\alpha\left[\alpha^{-1}\left({ }_{N} \sum_{k=1}^{n} a_{k}^{p_{N}}\right)\right] \dot{+} \alpha\left[\alpha^{-1}\left({ }_{N} \sum_{k=1}^{n} b_{k}^{p_{N}}\right)\right] \\
& =\sqrt[p]{\sum_{N=1}^{n} a_{k}^{p_{N}}} \dot{+}{\sqrt[p]{N} \sum_{k=1}^{n} b_{k}^{p_{N}}}^{N}
\end{aligned}
$$

which completes the proof.

Definition 3.4 Let $X$ be a non-empty set and $d_{N}: X \times X \rightarrow \mathbb{R}^{+}(N)$ be a function such that for all $x, y, z \in X$, the following non-Newtonian metric axioms hold:

(NM1) $d_{N}(x, y)=1$ if and only if $x=y$,

(NM2) $d_{N}(x, y)=d_{N}(y, x)$,

(NM3) $d_{N}(x, y) \dot{\leq} d_{N}(x, z) \dot{+} d_{N}(z, y)$. 
Then the pair $\left(X, d_{N}\right)$ and $d_{N}$ are called a non-Newtonian metric space and a nonNewtonian metric on $X$, respectively.

Corollary $3.5\left(\mathbb{R}(N), d_{N}\right)$ is a non-Newtonian metric space.

Proof Define the non-Newtonian metric $d_{N}$ on $\mathbb{R}(N)$ by $d_{N}(x, y)=|x \ominus y|_{N}(x, y \in \mathbb{R}(N))$. Then it is immediate for any $u, x, y \in \mathbb{R}(N)$ that

(NM1) It is easy to see that

$$
\begin{aligned}
d_{N}(u, x) & =|u \dot{-x}|_{N}=\alpha(0) \\
& \Leftrightarrow \alpha\left\{\left|\alpha^{-1}(u)-\alpha^{-1}(x)\right|\right\}=\alpha(0) \\
& \Leftrightarrow \alpha^{-1}(u)=\alpha^{-1}(x) \\
& \Leftrightarrow u=x .
\end{aligned}
$$

(NM2) One can obtain that

$$
\begin{aligned}
d_{N}(u, x) & =\alpha\left\{\left|\alpha^{-1}(u)-\alpha^{-1}(x)\right|\right\} \\
& =\alpha\left\{\left|\alpha^{-1}(x)-\alpha^{-1}(u)\right|\right\} \\
& =d_{N}(x, u) .
\end{aligned}
$$

(NM3) A direct calculation leads us to

$$
\begin{aligned}
d_{N}(u, y) & =|u \dot{-} y|_{N}=\alpha\left\{\left|\alpha^{-1}(u)-\alpha^{-1}(y)\right|\right\} \\
& =\alpha\left\{\left|\alpha^{-1}(u)-\alpha^{-1}(x)+\alpha^{-1}(x)-\alpha^{-1}(y)\right|\right\} \\
& \dot{\leq} \alpha\left\{\left|\alpha^{-1}(u)-\alpha^{-1}(x)\right|\right\} \dot{+} \alpha\left\{\left|\alpha^{-1}(x)-\alpha^{-1}(y)\right|\right\} \\
& =|u \dot{-} x|_{N} \dot{+}|x \dot{-} y|_{N} \\
& =d_{N}(u, x) \dot{+} d_{N}(x, y) .
\end{aligned}
$$

This is what we wished to show.

Theorem 3.6 n-dimensional non-Newtonian Euclidian space $\mathbb{R}^{n}(N)$, consisting of all ordered $n$-tuples of non-Newtonian real numbers, is a metric space with the metric $d_{N}$ defined by

$$
d_{N}(x, y)={\sqrt{\sum_{k=1}^{n}\left(x_{k} \dot{-} y_{k}\right)^{2}}}^{N}=\alpha\left\{\sqrt{\sum_{k=1}^{n}\left[\alpha^{-1}\left(x_{k}\right)-\alpha^{-1}\left(y_{k}\right)\right]^{2}}\right\},
$$

where $x=\left(x_{1}, x_{2}, \ldots, x_{n}\right), y=\left(y_{1}, y_{2}, \ldots, y_{n}\right) \in \mathbb{R}^{n}(N)$.

Proof Let $x=\left(x_{1}, x_{2}, \ldots, x_{n}\right), y=\left(y_{1}, y_{2}, \ldots, y_{n}\right)$ and $z=\left(z_{1}, z_{2}, \ldots, z_{n}\right) \in \mathbb{R}^{n}(N)$. Then (NM1) and (NM2) are obvious. To prove (NM3), we use Minkowski's inequality in NC with $p=2$, 
the Cauchy-Schwarz inequality in NC. Therefore, we see that

$$
\begin{aligned}
d_{N}(x, y) & ={\sqrt{{ }_{N} \sum_{k=1}^{n}\left(x_{k} \dot{-} y_{k}\right)^{2_{N}}}}^{N}={\sqrt{\sum_{N} \sum_{k=1}^{n}\left(x_{k} \dot{-} z_{k} \dot{+} z_{k} \dot{-} y_{k}\right)^{2_{N}}}}^{N} \\
& \dot{\leq}{\sqrt{N} \sum_{k=1}^{n}\left(x_{k} \dot{-} z_{k}\right)^{2_{N}}}^{N}+{\sqrt{{ }_{N} \sum_{k=1}^{n}\left(z_{k} \dot{-} y_{k}\right)^{2_{N}}}}^{N} \\
& =d_{N}(x, z) \dot{+} d_{N}(z, y),
\end{aligned}
$$

which completes the proof.

Now, we define the notion of norm in the sense of non-Newtonian calculus.

Definition 3.7 Let $X$ be a vector space over the field $\mathbb{R}(N)$ and $\|\cdot\|_{N}$ be a function from $X$ to $\mathbb{R}^{+}(N)$ satisfying the following non-Newtonian norm axioms: For $x, y \in X$ and $\alpha \in$ $\mathbb{R}(N)$,

(NN1) $\|x\|_{N}=\dot{0} \Leftrightarrow x=\dot{0}$,

(NN2) $\|\alpha \dot{\times} x\|_{N}=|\alpha|_{N} \dot{\times}\|x\|_{N}$,

(NN3) $\|x \dot{+} y\|_{N} \dot{\leq}\|x\|_{N} \dot{+}\|y\|_{N}$.

Then $\left(X,\|\cdot\|_{N}\right)$ is said to be a non-Newtonian normed space. It is trivial that a nonNewtonian norm $\|\cdot\|_{N}$ on $X$ defines a non-Newtonian metric $d_{N}$ on $X$ which is given by

$$
d_{N}(x, y)=\|x \dot{-} y\|_{N} \quad(x, y \in X)
$$

and is called the non-Newtonian metric induced by the non-Newtonian norm.

\section{Convergence and completeness in (NC)}

In this section, we emphasize some properties of convergent sequences in a nonNewtonian metric space and completeness of $n$-dimensional non-Newtonian metric space $\mathbb{R}^{n}(N)$.

Definition 4.1 (Convergent sequence) A sequence $\left(x_{n}\right)$ in a metric space $X=\left(X, d_{N}\right)$ is said to be convergent if for every given $\varepsilon \dot{>} \dot{0}$, there exist an $n_{0}=n_{0}(\varepsilon) \in \mathbb{N}$ and $x \in X$ such that $d_{N}\left(x_{n}, x\right) \dot{<\varepsilon}$ for all $n>n_{0}$ and is denoted by ${ }^{N} \lim _{n \rightarrow \infty} x_{n}=x$ or $x_{n} \stackrel{N}{\rightarrow} x$ as $n \rightarrow \infty$.

Definition 4.2 (Cauchy sequence) A sequence $\left(x_{n}\right)$ in a non-Newtonian metric space $X=$ $\left(X, d_{N}\right)$ is said to be non-Newtonian Cauchy if for every $\varepsilon \dot{>0}$, there is an $n_{0}=n_{0}(\varepsilon) \in \mathbb{N}$ such that $d_{N}\left(x_{n}, x_{m}\right)<\varepsilon$ for all $m, n>n_{0}$.

The space $X$ is said to be non-Newtonian complete if every non-Newtonian Cauchy sequence in $X$ converges.

Theorem 4.3 Let $X=\left(X, d_{N}\right)$ be a non-Newtonian metric space. Then:

(a) A convergent sequence in $X$ is bounded and its limit is unique.

(b) A convergent sequence in $X$ is a Cauchy sequence in $X$. 
Proof (a) Suppose that $\left(x_{n}\right)$ is a convergent sequence in a non-Newtonian metric space $X$ with $x_{n} \stackrel{N}{\rightarrow} x$. Then taking $\varepsilon=\alpha(1)=\dot{1}$, we can find an $n_{0}$ such that $d_{N}\left(x_{n}, x\right) \dot{<}$ for all $n>n_{0}$. Hence, by the non-Newtonian triangle inequality, for all $n$, we have $d_{N}\left(x_{n}, x\right)<\dot{1}+a$, where $a=\max \left\{d_{N}\left(x_{0}, x\right), d_{N}\left(x_{1}, x\right), \ldots, d_{N}\left(x_{n_{0}}, x\right)\right\}$. This shows that $\left(x_{n}\right)$ is bounded.

Assume that $x_{n} \stackrel{N}{\rightarrow} x$ and $x_{n} \stackrel{N}{\rightarrow} z$ as $n \rightarrow \infty$. Then we obtain from (NM3) that

$$
\dot{0}=\alpha(0) \dot{\leq} d_{N}(x, z) \dot{\leq} d_{N}\left(x, x_{n}\right) \dot{+} d_{N}\left(x_{n}, z\right)
$$

which leads us to the result as $n \rightarrow \infty$ that $d_{N}(x, z)=\dot{0}$. This means with (NM1) that the non-Newtonian limit is unique.

(b) Suppose that $\left(x_{n}\right)$ is a convergent sequence with the limit $x$ in a non-Newtonian metric space $X=\left(X, d_{N}\right)$. Then for every $\varepsilon \dot{>0}$, there is an $n_{0}=n_{0}(\varepsilon) \in \mathbb{N}$ such that $d_{N}\left(x_{n}, x\right) \dot{<} \varepsilon / 2$ for all $n>n_{0}$. Therefore, combining the hypothesis with the non-Newtonian triangle inequality one can observe for every $\varepsilon \dot{>0}$, there is an $n_{0}=n_{0}(\varepsilon) \in \mathbb{N}$ such that

$$
d_{N}\left(x_{n}, x_{m}\right) \dot{\leq} d_{N}\left(x_{n}, x\right) \dot{+} d_{N}\left(x, x_{m}\right) \dot{<} \varepsilon
$$

for all $m, n>n_{0}$. Hence, $\left(x_{n}\right)$ is a non-Newtonian Cauchy sequence.

This completes the proof.

Now, we can give the theorem on the completeness of the metric space $\left(\mathbb{R}^{n}(N), d_{N}\right)$.

Theorem 4.4 $\left(\mathbb{R}^{n}(N), d_{N}\right)$ is complete.

Proof It is known by Theorem 3.6 that $d_{N}$ defined by (3.6) is a metric on $\mathbb{R}^{n}(N)$. Suppose that $\left(x_{m}\right)=\left\{x_{k}^{(m)}\right\}$ is a Cauchy in $\mathbb{R}^{n}(N)$, where $x_{m}=\left(x_{1}^{(m)}, x_{2}^{(m)}, x_{3}^{(m)}, \ldots, x_{n}^{(m)}\right)$ for each fixed $m \in \mathbb{N}$. Since $\left(x_{m}\right)$ is non-Newtonian Cauchy, for every $\varepsilon>\dot{0}$ there is an $n_{0} \in \mathbb{N}$ such that

$$
d_{N}(x, y)={\sqrt{\sum_{N}^{n}\left[x_{k}^{(m)}-x_{k}^{(r)}\right]^{2_{N}}}}^{N} \dot{<\varepsilon}
$$

for all $m, r>n_{0}$. By non-Newtonian squaring, we have for all $m, r>n_{0}$ and all $k \in$ $\{1,2,3, \ldots, n\}$ that

$$
\left[x_{k}^{(m)} \dot{-} x_{k}^{(r)}\right]^{2_{N}}<\varepsilon^{2_{N}}
$$

which implies that

$$
\left|x_{k}^{(m)} \dot{-} x_{k}^{(r)}\right|_{N} \dot{<\varepsilon}
$$

This shows that for each fixed $k \in\{1,2, \ldots, n\},\left(x_{k}^{(1)}, x_{k}^{(2)}, x_{k}^{(3)}, \ldots\right)$ is a convergent sequence with $x_{k}^{(m)} \stackrel{N}{\rightarrow} x_{k}$ as $m \rightarrow \infty$. Using these $n$ limits, we define $x=\left(x_{1}, x_{2}, \ldots, x_{n}\right)$ in $\mathbb{R}^{n}(N)$. From (4.1), letting $r \rightarrow \infty$ it is obtained that $d_{N}\left(x_{m}, x\right) \leq \varepsilon$ for all $m>n_{0}$ which shows that $\left(x_{m}\right)$ converges in $\mathbb{R}^{n}(N)$. Consequently, $\left(\mathbb{R}^{n}, d_{N}\right)$ is a complete metric space. 
It is trivial that $\mathbb{R}^{n}(N)$ is a vector space over $\mathbb{R}(N)$ with respect to the algebraic operations addition $(\dot{+})$ and scalar multiplication $(\dot{x})$ defined on $\mathbb{R}^{n}(N)$ as follows:

$$
\begin{aligned}
\dot{+}: \mathbb{R}^{n}(N) \times \mathbb{R}^{n}(N) & \longrightarrow \mathbb{R}^{n}(N) \\
(x, y) & \mapsto x \dot{+} y=\left(x_{1} \dot{+} y_{1}, x_{2} \dot{+} y_{2}, \ldots, x_{n} \dot{+} y_{n}\right), \\
\dot{\times}: \mathbb{R}(N) \times \mathbb{R}^{n}(N) & \longrightarrow \mathbb{R}^{n}(N) \\
(\alpha, x) & \longmapsto \alpha \dot{\times} x=\left(\alpha \dot{\times} x_{1}, \alpha \dot{\times} x_{2}, \ldots, \alpha \dot{\times} x_{n}\right),
\end{aligned}
$$

where $x=\left(x_{1}, x_{2}, \ldots, x_{n}\right), y=\left(y_{1}, y_{2}, \ldots, y_{n}\right) \in \mathbb{R}^{n}(N)$ and $\alpha \in \mathbb{R}(N)$.

Since $\mathbb{R}^{n}(N)$ is a complete metric space with the metric $d_{N}$ defined by (3.6) induced by the norm $\|\cdot\|_{N}$, as a direct consequence of Theorem 4.4, we have:

Corollary $4.5 \mathbb{R}^{n}(N)$ is a Banach space with the norm $\|\cdot\|_{N}$ defined by

$$
\|x\|_{N}=\sqrt{\sum_{N=1}^{n}\left|x_{k}\right|_{N}^{2_{N}}} ; \quad x=\left(x_{1}, x_{2}, \ldots, x_{n}\right) \in \mathbb{R}^{n}(N) .
$$

\section{Sequence spaces over the non-Newtonian real field}

In this section, we define the sets $\omega(N), \ell_{\infty}(N), c(N), c_{0}(N)$ and $\ell_{p}(N)$ of all, bounded, convergent, null and absolutely $p$-summable sequences over the non-Newtonian real field $\mathbb{R}(N)$ which correspond to the sets $\omega, \ell_{\infty}, c, c_{0}$ and $\ell_{p}$ over the real field $\mathbb{R}$, respectively. That is to say,

$$
\begin{aligned}
& \omega(N)=\left\{x=\left(x_{k}\right): x_{k} \in \mathbb{R}(N) \text { for all } k \in \mathbb{N}\right\}, \\
& \ell_{\infty}(N)=\left\{x=\left(x_{k}\right) \in \omega(N): \sup _{k \in \mathbb{N}}\left|x_{k}\right|_{N}<\infty\right\}, \\
& c(N)=\left\{x=\left(x_{k}\right) \in \omega(N): \exists l \in \mathbb{R}(N) \ni{ }^{N} \lim _{k \rightarrow \infty}\left|x_{k} \dot{-}\right|_{N}=\dot{0}\right\}, \\
& c_{0}(N)=\left\{x=\left(x_{k}\right) \in \omega(N):{ }_{k \rightarrow \infty}^{N}\left|x_{k}\right|_{N}=\dot{0}\right\}, \\
& \ell_{p}(N)=\left\{x=\left(x_{k}\right) \in \omega(N):\left.{ }_{N}^{\infty}\left|x_{k=1}^{\infty}\right| x_{N}\right|_{N}<\infty\right\} \quad(1 \leq p<\infty) .
\end{aligned}
$$

One can easily see that the set $\omega(N)$ forms a vector space over $\mathbb{R}(N)$ with respect to the algebraic operations (+) addition and scalar multiplication (.) defined on $\omega(N)$ as follows:

$$
\begin{aligned}
+: \omega(N) \times \omega(N) & \longrightarrow \omega(N) \\
(x, y) & \mapsto x+y=\left(x_{k} \dot{+} y_{k}\right) \\
\cdot: \mathbb{R}(N) \times \omega(N) & \longrightarrow \omega(N) \\
(\alpha, x) & \mapsto \alpha \cdot x=\left(\alpha \dot{\times} x_{k}\right),
\end{aligned}
$$

where $x=\left(x_{k}\right), y=\left(y_{k}\right) \in \omega(N)$ and $\alpha \in \mathbb{R}(N)$. It is not hard to show that the sets $\ell_{\infty}(N)$, $c(N), c_{0}(N)$ and $\ell_{p}(N)$ are the subspaces of the space $\omega(N)$. This means that $\ell_{\infty}(N), c(N)$, $c_{0}(N)$ and $\ell_{p}(N)$ are classical sequence spaces over the field $\mathbb{R}(N)$. 
Theorem 5.1 Let $\dot{1}=\alpha(1), \dot{2}=\alpha(2)$. Define $d_{N}$ on the non-Newtonian space $\omega(N)$ by

$$
d_{N}(x, y)=\sum_{N=1}^{\infty} \frac{\left|x_{k} \dot{-} y_{k}\right|_{N}}{\dot{2}^{k_{N}} \dot{x}\left(\dot{\mathrm{i}} \dot{+}\left|x_{k} \dot{-} y_{k}\right|_{N}\right)} N
$$

where $x=\left(x_{k}\right), y=\left(y_{k}\right) \in \omega(N)$. Then $\left(\omega(N), d_{N}\right)$ is a metric space.

Proof Since the axioms (NM1) and (NM2) easily hold, we omit the details. The inequality (3.3) implies that

$$
\begin{aligned}
\sum_{N} \sum_{k=1}^{n} \frac{\left|x_{k} \dot{-} y_{k}\right|_{N}}{\dot{2}^{k_{N}} \dot{x}\left(\dot{1} \dot{1}\left|x_{k} \dot{-} y_{k}\right|_{N}\right)} N \leq & \sum_{N} \sum_{k=1}^{n} \frac{\left|x_{k} \dot{-} z_{k}\right|_{N}}{\dot{2}^{k_{N}} \dot{x}\left(\dot{1} \dot{\dot{1}}\left|x_{k} \dot{-} z_{k}\right|_{N}\right)} N \\
& \dot{+} \sum_{N} \sum_{k=1}^{n} \frac{\left|z_{k} \dot{-} y_{k}\right|_{N}}{\dot{2}^{k_{N}} \dot{x}\left(\dot{\mathrm{i}} \dot{+}\left|z_{k} \dot{-} y_{k}\right|_{N}\right)} N
\end{aligned}
$$

holds for all $n \in \mathbb{N}$. Additionally, since the inequalities

$$
\begin{gathered}
\frac{\left|x_{k}-y_{k}\right|_{N}}{\dot{2}^{k_{N}} \dot{x}\left(\dot{1}+\left|x_{k} \dot{-} y_{k}\right|_{N}\right)} N \dot{\leq} \frac{\dot{1}}{\dot{2}^{k_{N}}} N, \\
\frac{\left|x_{k}-z_{k}\right|_{N}}{\dot{2}^{k_{N}} \dot{\times}\left(1+\left|x_{k} \dot{-} z_{k}\right|_{N}\right)} N \dot{\leq} \frac{\dot{1}}{\dot{2}^{k_{N}}} N, \\
\frac{\left|z_{k} \dot{-} y_{k}\right|_{N}}{\dot{2}^{k_{N}} \dot{\times}\left(1+\left|x_{k} \dot{-} z_{k}\right|_{N}\right)} N \dot{\leq} \frac{\dot{1}}{\dot{2}^{k_{N}}} N
\end{gathered}
$$

hold for all $k \in \mathbb{N}$, the comparison test implies the convergence of the series

$$
\begin{aligned}
& \sum_{N=1}^{\infty} \frac{\left|x_{k} \dot{-} y_{k}\right|_{N}}{\dot{2}^{k_{N}} \dot{x}\left(\dot{\mathrm{i}} \dot{+}\left|x_{k} \dot{-} y_{k}\right|_{N}\right)} N, \\
& \sum_{N=1}^{\infty} \frac{\left|x_{k} \dot{-} z_{k}\right|_{N}}{\dot{2}^{k_{N}} \dot{x}\left(\dot{\mathrm{i}} \dot{+}\left|x_{k} \dot{-} z_{k}\right|_{N}\right)} N, \\
& \sum_{N=1}^{\infty} \frac{\left|z_{k} \dot{-} y_{k}\right|_{N}}{\dot{2}^{k_{N}} \dot{x}\left(\dot{\mathrm{i}} \dot{+}\left|z_{k} \dot{-} y_{k}\right|_{N}\right)} N .
\end{aligned}
$$

Therefore, by letting $n \rightarrow \infty$ in (5.1),

$$
\begin{aligned}
& d_{N}(x, y)=\sum_{N}^{\infty} \frac{\left|x_{k} \dot{-} y_{k}\right|_{N}}{\dot{2}^{k_{N}} \dot{x}\left(\dot{1} \dot{1}\left|x_{k} \dot{-} y_{k}\right|_{N}\right)} N \\
& \dot{\leq} \sum_{N=1}^{\infty} \frac{\left|x_{k} \dot{-} z_{k}\right|_{N}}{\dot{2}^{k_{N}} \dot{x}\left(\dot{1} \dot{1}\left|x_{k} \dot{-} z_{k}\right|_{N}\right)} N \dot{+} \sum_{N}^{\infty} \frac{\left|z_{k} \dot{-} y_{k}\right|_{N}}{2^{k_{N}} \dot{x}\left(\dot{\mathrm{i}} \dot{+}\left|z_{k} \dot{-} y_{k}\right|_{N}\right)} N \\
& =d_{N}(x, z) \dot{+} d_{N}(z, y),
\end{aligned}
$$

i.e., (NM3) holds. This step completes the proof. 
Theorem 5.2 Let $\lambda$ denote any of the spaces $\ell_{\infty}, c$ and $c_{0}$, and $x=\left(x_{k}\right), y=\left(y_{k}\right) \in \lambda(N)$. Define $d_{N_{\infty}}$ on the space $\lambda(N)$ by $d_{N_{\infty}}(x, y)=\sup _{k \in \mathbb{N}}\left|x_{k} \dot{-} y_{k}\right|_{N}$. Then $\left(\lambda(N), d_{N_{\infty}}\right)$ is a complete metric space.

Proof Since the proof is similar for the spaces $c(N)$ and $c_{0}(N)$, we prove the theorem only for the space $\ell_{\infty}(N)$. Let $x=\left(x_{k}\right), y=\left(y_{k}\right), z=\left(z_{k}\right) \in \ell_{\infty}(N)$. Then:

(i) Suppose that $d_{N_{\infty}}(x, y)=\dot{0}$. This implies for all $k \in \mathbb{N}$ that $x_{k} \dot{-} y_{k}=\dot{0}$, i.e., $x=y$.

Conversely, let $x=y$. Then we have $x_{k} \dot{-} y_{k}=\dot{0}$ for all $k \in \mathbb{N}$ which yields that

$d_{N_{\infty}}(x, y)=\dot{0}$.

That is to say, (NM1) holds.

(ii) It is immediate that $d_{N_{\infty}}(x, y)=d_{N_{\infty}}(y, x)$, i.e., (NM2) holds.

(iii) By taking into account the triangle inequality in (3.1), we see that

$$
\begin{aligned}
d_{N_{\infty}}(x, y) & =\sup _{k \in \mathbb{N}}\left|x_{k} \dot{-} y_{k}\right|_{N} \\
& =\sup _{k \in \mathbb{N}}\left|x_{k} \dot{-} y_{k} \dot{+} z_{k} \dot{-} z_{k}\right|_{N} \\
& \leq \sup _{k \in \mathbb{N}}\left|x_{k} \dot{-} z_{k}\right|_{N}+\sup _{k \in \mathbb{N}}\left|y_{k} \dot{-} z_{k}\right|_{N} \\
& =d_{N_{\infty}}(x, z) \dot{+} d_{N_{\infty}}(z, y) .
\end{aligned}
$$

Hence, (NM3) holds.

Since the axioms (NM1)-(NM3) are satisfied, $\left(\ell_{\infty}(N), d_{N_{\infty}}\right)$ is a metric space.

It remains to prove the completeness of the space $\ell_{\infty}(N)$. Let $\left(x^{m}\right)$ be any sequence in the space $\ell_{\infty}(N)$, where $x^{m}=\left\{x_{1}^{(m)}, x_{2}^{(m)}, \ldots\right\}$. Then for any $\varepsilon \dot{>}$, there is an $n_{0}$ such that for all $m, n>n_{0}$,

$$
d_{N_{\infty}}\left(x^{m}, x^{n}\right)=\sup _{k \in \mathbb{N}}\left|x_{k}^{(m)} \dot{-} x_{k}^{(n)}\right|_{N} \dot{<\varepsilon}
$$

A fortiori, for every fixed $k \in \mathbb{N}$ and for all $m, n>n_{0}$,

$$
\left|x_{k}^{(m)} \dot{-} x_{k}^{(n)}\right|_{N} \dot{<\varepsilon}
$$

Hence, for every fixed $k \in \mathbb{N}$, the sequence $x_{m}=\left\{x_{k}^{(1)}, x_{k}^{(2)}, \ldots\right\}$ is a Cauchy sequence of nonNewtonian real numbers. Since $\mathbb{R}(N)$ is a complete metric space in the non-Newtonian sense by Theorem 4.4 with $n=1$, it converges, say $x_{k}^{(m)} \stackrel{N}{\rightarrow} x_{k}$ as $m \rightarrow \infty$. Using these infinitely many limits $x_{1}, x_{2}, \ldots$, we define $x=\left(x_{1}, x_{2}, \ldots\right)$ and show that $x \in \ell_{\infty}(N)$. From (5.3) letting $n \rightarrow \infty$ and $m>n_{0}$ we have

$$
\left|x_{k}^{(m)} \dot{-} x_{k}\right|_{N} \dot{\leq} \varepsilon
$$

Since $\left(x^{m}\right)=\left\{x_{k}^{(m)}\right\} \in \ell_{\infty}(N)$, there is a real number $K_{m}$ such that $\left|x_{k}^{(m)}\right|_{N} \dot{\leq} K_{m}$ for all $k \in \mathbb{N}$. Thus, (5.4) together with the triangle inequality in (3.1) gives for $m>n_{0}$ that

$$
\left|x_{k}\right|_{N} \dot{\leq}\left|x_{k} \dot{-} x_{k}^{(m)}\right|_{N}+\left|x_{k}^{(m)}\right|_{N} \dot{\leq} \varepsilon+K_{m}
$$

It is clear that (5.5) holds for every $k \in \mathbb{N}$ whose right-hand side does not involve $k$. Hence, $\left(x_{k}\right)$ is a bounded sequence of non-Newtonian real numbers, that is, $x=\left(x_{k}\right) \in \ell_{\infty}(N)$. Also 
from (5.4) we obtain for $m>n_{0}$ that

$$
d_{N_{\infty}}\left(x^{m}, x\right)=\sup _{k \in \mathbb{N}}\left|x_{k}^{(m)} \dot{-} x_{k}\right|_{N} \dot{\leq} \varepsilon
$$

This shows that $x^{m} \stackrel{N}{\rightarrow} x$ as $m \rightarrow \infty$. Since $\left(x^{m}\right)$ was an arbitrary non-Newtonian Cauchy sequence, $\ell_{\infty}(N)$ is complete.

Since it is known by Theorem 5.2 that the spaces $\ell_{\infty}(N), c(N)$ and $c_{0}(N)$ are complete metric spaces with the metric $d_{N_{\infty}}$ induced by the norm $\|\cdot\|_{N_{\infty}}$ defined by

$$
\|x\|_{N_{\infty}}=\sup _{k \in \mathbb{N}}\left|x_{k}\right|_{N} ; \quad x=\left(x_{k}\right) \in \lambda(N), \lambda \in\left\{\ell_{\infty}, c, c_{0}\right\},
$$

we have:

Corollary 5.3 The spaces $\ell_{\infty}(N), c(N)$ and $c_{0}(N)$ are Banach spaces with the norm $\|\cdot\|_{N_{\infty}}$ defined by (5.6).

To avoid undue repetition in the statements, we give the final theorem without proof concerning the complete metric space $\ell_{p}(N)$.

Theorem 5.4 Define $d_{N_{p}}$ on the non-Newtonian space $\ell_{p}(N)$ by $d_{N_{p}}(x, y)=\left({ }_{N} \sum_{k=0}^{\infty} \mid x_{k} \dot{-}\right.$ $\left.\left.y_{k}\right|_{N} ^{p_{N}}\right)^{(1 / p)_{N}}$, where $x=\left(x_{k}\right), y=\left(y_{k}\right) \in \ell_{p}(N)$ and $p \geq 1$. Then $\left(\ell_{p}(N), d_{N_{p}}\right)$ is a complete metric space.

Since it is known by Theorem 5.4 that the space $\ell_{p}(N)$ is a complete metric space with the metric $d_{N_{p}}$ induced by the norm $\|\cdot\|_{N_{p}}$ defined by

$$
\|x\|_{N_{p}}=\left(\sum_{N=0}^{\infty}\left|x_{k}\right|_{N}^{p_{N}}\right)^{(1 / p)_{N}} \quad\left(x=\left(x_{k}\right) \in \ell_{p}(N), p \geq 1\right),
$$

we have:

Corollary 5.5 The space $\ell_{p}(N)$ is a Banach space with the norm $\|\cdot\|_{N_{p}}$ defined by (5.7).

\section{Conclusion}

One of the purposes of this work is to extend non-Newtonian calculus to non-Newtonian real calculus for dealing with real valued functions. Some of the analogies between (CC) and (NC) are demonstrated by theoretical examples. Some important inequalities, such as non-Newtonian triangle, non-Newtonian Minkowski and some other inequalities which are frequently used in (NC) are proved. We derive classical sequence spaces in the sense of non-Newtonian calculus and try to understand their structure of being a non-Newtonian vector space. Generally, we work on vector spaces which concern physics and computing. There are lots of techniques that have been developed in the sense of (CC). If (NC) is employed together with $(\mathrm{CC})$ in the formulations, then many of the complicated phenomena in physics or engineering may be analyzed more easily. 
Quite recently, Talo and Başar have studied the certain sets of sequences of fuzzy numbers and introduced the classical sets $\ell_{\infty}(F), c(F), c_{0}(F)$ and $\ell_{p}(F)$ consisting of bounded, convergent, null and absolutely $p$-summable sequences of fuzzy numbers in [5]. Next, they have defined the $\alpha-, \beta$ - and $\gamma$-duals of a set of sequences of fuzzy numbers, and gave the duals of the classical sets of sequences of fuzzy numbers together with the characterization of the classes of infinite matrices of fuzzy numbers transforming one of the classical sets into another. Following Bashirov et al. [2] and Uzer [3], we have given the corresponding results for non-Newtonian calculus to the results obtained for fuzzy valued sequences in Talo and Başar [5] as a beginning. As a natural continuation of this paper, we should record that it is meaningful to define the $\alpha$-, $\beta$ - and $\gamma$-duals of a space of sequences of nonNewtonian real entries and to determine the duals of classical spaces $\ell_{\infty}(N), c(N), c_{0}(N)$ and $\ell_{p}(N)$ together with the characterization of matrix transformations between the classical sequence spaces over the non-Newtonian real field $\mathbb{R}(N)$. Further, one can obtain similar results by using another type of calculus instead of non-Newtonian calculus.

It is natural to expect that the Banach spaces $\ell_{\infty}(N), c(N), c_{0}(N)$ and $\ell_{p}(N)$ can be extended to the complete paranormed sequence spaces $\ell_{\infty}(p, N), c(p, N), c_{0}(p, N)$ and $\ell(p, N)$ so as the Maddox's spaces $\ell_{\infty}(p), c(p), c_{0}(p)$ and $\ell(p)$ ( $c f$. Maddox [6, 7], Nakano [8] and Simons [9]) are derived on the real or complex field from the classical sequence spaces $\ell_{\infty}$, $c, c_{0}$ and $\ell_{p}$, respectively. We note from now on that our next paper will be devoted to the investigation of this subject.

Following Grossman and Katz [1], Türkmen and Başar [4] constructed the field $\mathbb{C}(G)$ of geometric complex numbers and gave the triangle and Minkowski's inequalities in the sense of geometric calculus. Later, they respectively defined the classical sets $w(G), \ell_{\infty}(G)$, $c(G), c_{0}(G)$ and $\ell_{p}(G)$ of all, bounded, convergent, null and $p$-absolutely summable sequences of geometric complex entries and showed that each of the set forms a complete vector space on the field $\mathbb{C}(G)$. Of course, one can determine the $\alpha$-, $\beta$ - and $\gamma$-duals of the spaces $\ell_{\infty}(G), c(G), c_{0}(G)$ and $\ell_{p}(G)$ and characterize some classes of matrix transformation between these spaces in the sense of geometric calculus.

\section{Competing interests}

The authors declare that they have no competing interests.

Authors' contributions

All the authors contributed equally and significantly in writing this paper. All authors read and approved the final manuscript.

\section{Author details}

${ }^{1}$ Department of Mathematical Engineering, Yıldız Technical University, Davutpaşa Campus, Esenler, İstanbul, 80750 Turkey. ${ }^{2}$ Department of Mathematics, Faculty of Arts and Sciences, Fatih University, The Hadımköy Campus, Büyükçekmece, İstanbul, 34500, Turkey.

Received: 31 May 2012 Accepted: 20 September 2012 Published: 11 October 2012

\section{References}

1. Grossman, M, Katz, R: Non-Newtonian Calculus. Lee Press, Pigeon Cove (1972) (Lowell Technological Institute)

2. Bashirov, AE, Kurpınar, EM, Özyapıcı, A: Multiplicative calculus and its applications. J. Math. Anal. Appl. 337, 36-48 (2008)

3. Uzer, A: Multiplicative type complex calculus as an alternative to the classical calculus. Comput. Math. Appl. 60, 2725-2737 (2010)

4. Türkmen, C, Başar, F: Some basic results on the sets of sequences with geometric calculus. AIP Conf. Proc. 1470, 95-98 (2012)

5. Talo, Ö, Başar, F: Determination of the duals of classical sets of sequences of fuzzy numbers and related matrix transformations. Comput. Math. Appl. 58, 717-733 (2009)

6. Maddox, IJ: Paranormed sequence spaces generated by infinite matrices. Math. Proc. Camb. Philos. Soc. 64, 335-340 (1968) 
7. Maddox, IJ: Spaces of strongly summable sequences. Q. J. Math. 18, 345-355 (1967)

8. Nakano, H: Modulared sequence spaces. Proc. Jpn. Acad. 27(2), 508-512 (1951)

9. Simons, S: The sequence spaces $\ell\left(p_{v}\right)$ and $m\left(p_{v}\right)$. Proc. Lond. Math. Soc. 15, 422-436 (1965)

doi:10.1186/1029-242X-2012-228

Cite this article as: Çakmak and Başar: Some new results on sequence spaces with respect to non-Newtonian calculus. Journal of Inequalities and Applications 2012 2012:228.

Submit your manuscript to a SpringerOpen ${ }^{\circ}$ journal and benefit from:

- Convenient online submission

- Rigorous peer review

- Immediate publication on acceptance

- Open access: articles freely available online

- High visibility within the field

- Retaining the copyright to your article

Submit your next manuscript at $>$ springeropen.com 